\title{
Three-dimensional Finite Element Modeling of Skeletal Muscle Using a Two-domain Approach: Linked Fiber-Matrix Mesh Model
}

\author{
Can A. Yucesoy ${ }^{1}$, Bart H.F.J.M. Koopman ${ }^{1}$, Peter A. Huijing ${ }^{1,2}$ and Henk J. Grootenboer ${ }^{1}$ \\ ${ }^{1}$ Institute for Biomedical Technology, Department of Mechanical Engineering, \\ University of Twente, Enschede, The Netherlands \\ ${ }^{2}$ Faculty of Human Movement Sciences, Vrije Universiteit, Amsterdam, The Netherlands
}

\section{Introduction}

Skeletal muscle tissue is composed of activatable muscle fibers and a passive matrix of connective tissues that houses them. The intra-sarcomeric cytoskeleton is composed of several proteins and anchors the myofilaments (Berthier and Blaineau, 1997). Muscle can be represented as an extensive 3D set of endomysial tunnels within which the myofibers operate (Trotter and Purslow, 1992). Although the myotendinous junction is often regarded the major or only site of force transmission, a relatively recent perspective includes also the costameres as a junctional domain. The multimolecular cytoskeletal complexes extending over intracytoplasmic to subsarcolemmal and transmembranous levels provide a route of force transmission to the extracellular matrix (Berthier and Blaineau, 1997). The mechanism of force transmission from the full perimeter surface of myofibrils on the extracellular connective tissue matrix was evident in a number of experimental studies (Huijing, 1999a; Huijing, 1999b; Huijing et al., 1998; Street, 1983; Trotter and Purslow, 1992).

In previous finite element modeling the passive and active properties of muscle were lumped in one element. For a realistic approach, skeletal muscle should be considered in two separate domains: (1) the intracellular domain and (2) extracellular matrix domain. The goal of the present work is to develop a model in which these domains are represented by two separate but linked meshes. An obvious goal of this way of modeling is to allow force transmission between these domains and assess the interaction between them. The model is presently applied to study the muscular dystrophies in which diseases, the system of trans-sarcolemmal connections is affected.

\section{Methods}

Two 3D-elements with eight nodes and linear interpolation functions were developed, and were introduced into ANSYS 5.5.1 FEM software as user-defined elements. One of these elements represents the extracellular matrix, which includes the basal lamina as well as connective tissue components such as endomysium and perimysium (extracellular matrix element). The second one accounts for the muscle fibers, (myofiber element). Within the biological context, one muscle element is defined to represent a segment of a bundle of muscle fibers with identical material properties, its connective tissues and the links between them. This is realized as a linked system of extracellular matrix and myofiber elements (Fig.1a). In the linked fiber-matrix mesh model, the extracellular matrix domain is represented by a mesh of extracellular matrix elements and is referred to as matrix mesh. In the same space, a separate mesh of myofiber elements is built to account for the intracellular domain and is named fiber mesh. The two meshes are rigidly connected to single layers of aponeurosis elements at the myotendinous connection sites and are linked elastically at the intermediate nodes. The elastic links are adjustable in stiffness to allow manipulation of the interaction between the two domains. The links are represented by the spring element COMBIN39, one of the standard elements of ANSYS 5.5.1, which is set to be longitudinal and have linear stiffness characteristics. To represent the aponeurosis another standard element, HYPER58 is used. The geometry of the muscle model (Fig.1b) is presently defined by the contour of a longitudinal slice of the isolated rat medial gastrocnemius muscle at the middle of the muscle belly. Three muscle elements in series and six in parallel fill the outline of this slice. This means that the collection of these muscle elements in series represent a big muscle fascicle. All aponeurosis elements have identical properties but variable thickness in the fiber-cross fiber plane such 
that the most proximal and most distal ends of the aponeurosis elements representing the muscles' tendons are thicker. This way an increasing stiffness towards the tendons is introduced.
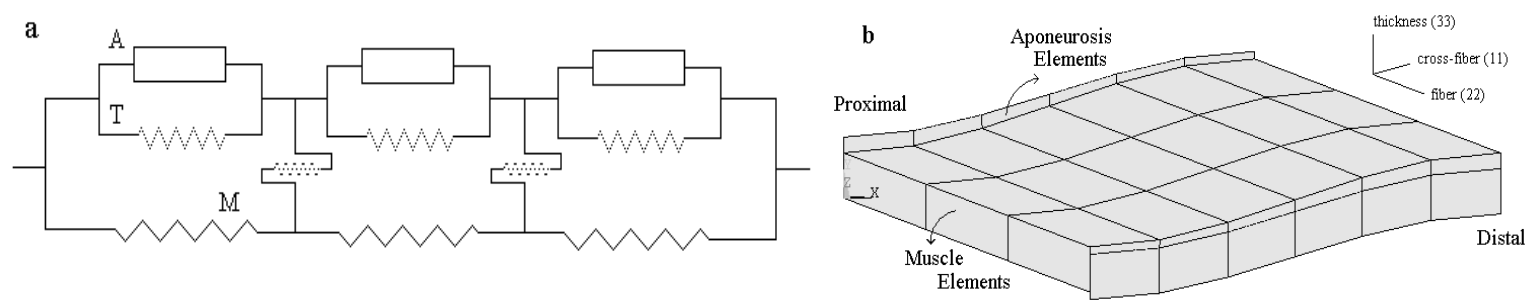

Fig.1. (a) 1D-Schematic representation of an arrangement of muscle elements. Intracellular domain which is composed of active contractile elements (A) and intracellular passive cytoskeleton (T), is linked to the extracellular matrix domain (M) elastically. (b) Geometry of the muscle model. The geometry is defined by the contour of a longitudinal slice of the isolated rat medial gastrocnemius muscle belly. The initial muscle length that is the distance between the most proximal and distal ends is $30.3 \mathrm{~mm}$. A 3D local coordinate system representing the fiber direction, cross-fiber (direction perpendicular to the fiber direction) and thickness directions is used for the analysis and presentation of the results.

In order to model possible effects of muscular dystrophies, the linking stiffness was manipulated at selected locations within the muscle such that at 8 of the total 28 nodes links were made much more compliant then the highly stiff remainder links. Decreasing the linking stiffness at these nodal locations represents the missing or altered connection between the muscle fibers and the extracellular matrix all along the full length of fibers of the fascicle.

\section{Results and Discussion}

Three sets of locations which are referred to as $\mathrm{A}, \mathrm{B}$ and $\mathrm{C}$ were considered. In these cases, all the links were highly stiff except for the ones at the indicated locations (Fig.2), which are much more compliant. A comparison of modeled muscle length-force characteristics with the stiff and compliant links is shown in Fig.2. For the low length range not much difference was found as a result of manipulation of linking stiffness. In contrast, for compliant links at certain intramuscular locations, the high length range yielded sharp drops in force accompanying very small changes of isometric length. This drop is enhanced, as the compliant link locations are more towards the middle of the muscle belly. In contrast, for compliant link locations at the periphery of the muscle, also at high lengths little effect is seen.
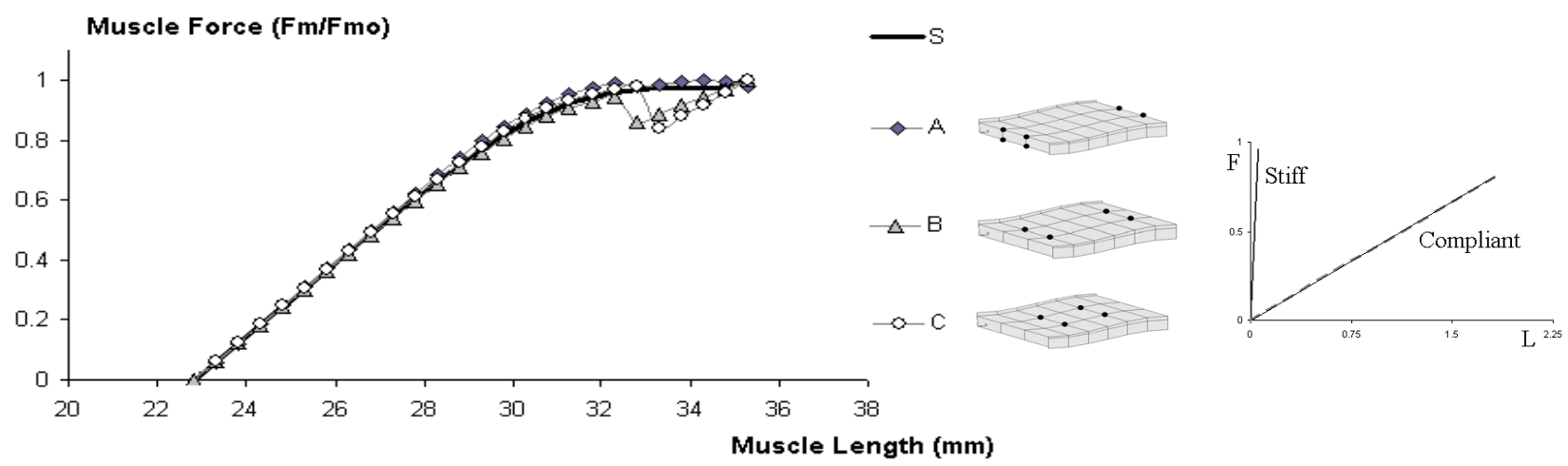

Fig.2. The muscle length-total force curves. $\mathrm{S}$ is the control case with all links being highly stiff. For the other three cases, the links at the nodes of intramuscular locations $\mathrm{A}, \mathrm{B}$ and $\mathrm{C}$ are compliant. The muscle force $(\mathrm{Fm})$ is normalized for optimum force (Fmo). Initial muscle length is $30.3 \mathrm{~mm}$. Comparison of the normalized length-force curves of the stiff and compliant links as a measure of their relative stiffness is given in the right hand side. 
The reason for the force drop can be understood from the stress and strain distributions in the fiber mesh (Fig.3). For intervention at location $C$, the total fiber direction stress $\left(\sigma_{22 \mathrm{f}}\right)$ is compared for two muscle lengths: The length just below which the force drop occurs (Fig.3a) and the length of the force drop (Fig.3b). A sizable decrease in the overall stress is apparent. At the lower of these muscle lengths, the $\sigma_{22 \mathrm{f}}$ values approximate unity throughout the fiber mesh (Fig 3a), but it is up to $80 \%$ lower locally at the higher length (Fig.3b). Within the fiber mesh, $\sigma_{22 \mathrm{f}}$ is a function of the fiber direction strain, $\varepsilon_{22}$. Distribution of $\varepsilon_{22}$ is highly uniform at very low values at the length just below the force drop length (Fig.3c). In contrast, the fiber mesh exibits very high strains near the specific locations of the compliant links at the force drop length (Fig.3d). These locally high strains reach values over unity for which very small or zero active stresses are calculated. Also the high values of negative strains observed in the fiber mesh (up to -0.27), leads to low values of stress in the fiber direction. This sharp decrease in stress at higher length generates the force drop.

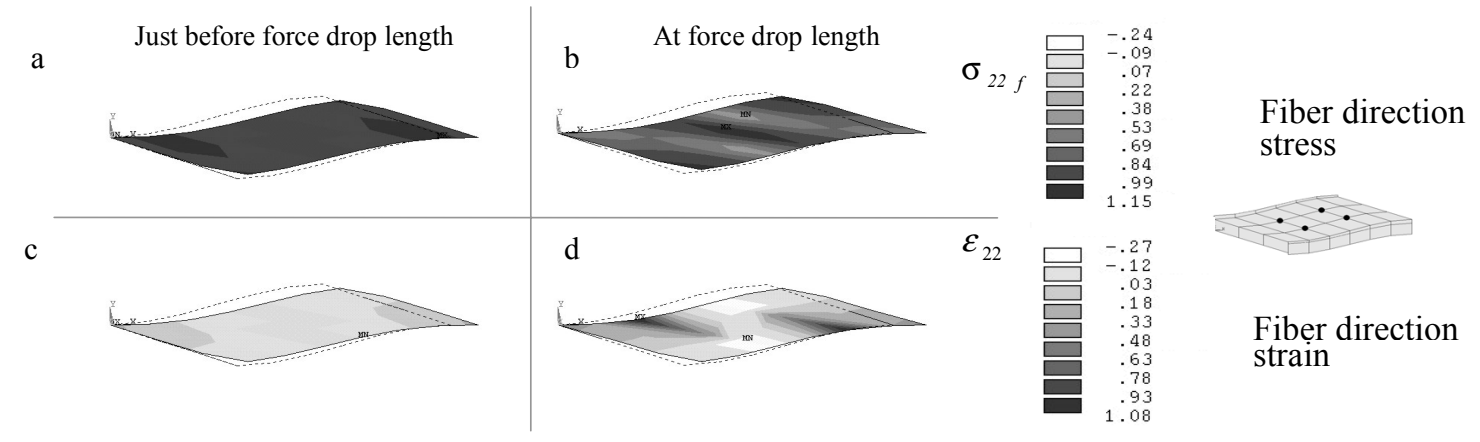

Fig.3. The total stress and strain distributions for the fiber mesh, in the fiber direction at high muscle lengths, with compliant links. (a) and (b) The total stress is the sum of the active stress of the contractile elements and the stress due to the passive intrasarcomeric cytoskeleton. (c) and (d) Strain distributions of the fiber mesh for the fiber (22) direction. Stress and strain distributions are shown for two muscle lengths equal to $32.8 \mathrm{~mm}$ and $33.3 \mathrm{~mm}$ respectively. The higher length is the length at which the force drop was found and the lower length is just below it. Results are shown for the case with compliant links located intramuscularly (case $\mathrm{C}$, marked with dots, in inset). The dotted line contour indicates muscle geometry at the initial length.

The application of the model demonstrates the role of extracellular matrix for a muscle in sustaining its physiological condition. In the pathological case, missing or altered trans-sarcolemmal connections to the muscle fibers, as associated with muscular dystrophies are encountered. The model results show that in the presence of such inadequate linking to the extracellular matrix, the myofibers are deformed beyond physiological limits due to lacking mechanical support and force transmission pathway by the extracellular matrix. Depending on the modeled muscles' geometry, the results indicate that a muscle with only myotendinous force transmission mechanism cannot function properly.

\section{References}

Berthier, C. and S. Blaineau (1997). "Supramolecular organization of the subsarcolemmal cytoskeleton of adult skeletal muscle fibers. A review." Biol Cell (7): 413-34.

Huijing, P. A. (1999a). "Muscle as a collagen fiber reinforced composite material: Force transmission in muscle and whole limbs." Journal of Biomechanics: 329-345.

Huijing, P. A. (1999b). "Muscular force transmission: A unified, dual or multiple sytem ? A review and some explorative experimental results." Archives of Physiology and Biochemistry (4): 292-311.

Huijing, P. A., G. C. Baan and G. Rebel (1998). "Non myo-tendinous force transmission in rat extensor digitorum longus muscle." Journal of Experimental Biology: 682-691.

Street, S. F. (1983). "Lateral transmission of tension in frog myofibers: a myofibrillar network and transverse cytoskeletal connections are possible transmitters." J Cell Physiol (3): 346-64.

Trotter, J. A. and P. P. Purslow (1992). "Functional morphology of the endomysium in series fibered muscles.” Journal of Morphology (2): 109-22. 